University of Nebraska - Lincoln

DigitalCommons@University of Nebraska - Lincoln

\title{
Predator-Dependent Functional Responses Alter the Coexistence and Indirect Effects among Prey that Share a Predator
}

Kyle E. Coblentz

University of Nebraska - Lincoln, kyle.coblentz@unl.edu

John P. DeLong

University of Nebraska - Lincoln, jpdelong@unl.edu

Follow this and additional works at: https://digitalcommons.unl.edu/bioscifacpub

Part of the Biology Commons, and the Ecology and Evolutionary Biology Commons

Coblentz, Kyle E. and DeLong, John P., "Predator-Dependent Functional Responses Alter the Coexistence and Indirect Effects among Prey that Share a Predator" (2020). Faculty Publications in the Biological Sciences. 826.

https://digitalcommons.unl.edu/bioscifacpub/826

This Article is brought to you for free and open access by the Papers in the Biological Sciences at DigitalCommons@University of Nebraska - Lincoln. It has been accepted for inclusion in Faculty Publications in the Biological Sciences by an authorized administrator of DigitalCommons@University of Nebraska - Lincoln. 


\title{
Predator-Dependent Functional Responses Alter the Coexistence and Indirect Effects among Prey that Share a Predator
}

\author{
Kyle E. Coblentz and John P. DeLong \\ School of Biological Sciences, University of Nebraska-Lincoln, Lincoln, Nebraska, USA \\ kyle.coblentz@unl.edu, https://orcid.org/0000-0002-0069-8491 \\ jpdelong@unl.edu, https://orcid.org/0000-0003-0558-8213
}

\begin{abstract}
Predator functional responses describe predator feeding rates as a function of prey abundance and are central to predator-prey theory. Despite ample evidence that functional responses also depend on predator abundance, theory incorporating predator-dependent functional responses has focused almost exclusively on specialist predator-prey pairs or linear food chains. This leaves a large gap in our knowledge as many predators feed on multiple prey, and in so doing, generate indirect effects among prey that can alter their coexistence. Here we investigate how predator-dependent functional responses in a one predator-two prey model alter the coexistence among prey and their net effects on one another. We use two different functional response forms (the Beddington-DeAngelis and Crowley-Martin functional responses) and consider situations in which the prey do not directly interact and in which they directly compete with one another. We find that predator dependence can facilitate, hinder, or have no effect on prey coexistence depending on whether prey compete directly and the role of predation in mediating coexistence among the prey in the absence of predator dependence. We also show that the negative net effects of prey on one another are generally weakened by predator dependence and can become positive under the Crowley-Martin functional response. Together, these results suggest that predator dependence may have widespread effects on ecological communities by altering the coexistence among prey species and the strength and signs of the interactions among them.
\end{abstract}

Keywords: Predator-prey interactions, Interaction strengths, Interference, Competition, Apparent competition, Apparent mutualism, Net effects, Press perturbations, Invasibility

\section{Introduction}

The predator functional response is central to predator-prey theory as it describes the feeding rates of predators on their prey (Solomon 1949, Holling 1959). Traditionally, predator-prey theory has focused on prey-dependent functional responses in which the feeding rates of predators are a function of only the density of prey available to predators (Holling 1959, Abrams and Ginzburg 2000). However, increasing evidence suggests that functional responses are generally predator-dependent; that is, the feeding rates of predators are functions of both predator and prey densities (Abrams and Ginzburg 2000, DeLong and Vasseur 2011, Arditi and Ginzburg 2012). In particular, predator-dependent functional responses generally model predator feeding rates as decreasing functions of predator densities due to processes such as predator interference or 'wasted time' interacting with other predators (Hassel and Varley 1969, Beddington 1975, DeAngelis et al. 1975, Crowley and Martin 1989, DeLong and Vasseur 2011). Theory has shown that the density dependent effect of predator densities on feeding rates can have important ecological consequences such as altering the stability and dynamics of predator-prey interactions and causing fundamental changes in food chain responses to enrichment (DeAngelis et al. 1975, Arditi and Ginzburg 1989, Arditi et al. 2004).
Despite the potential importance of predator-dependent functional responses for our understanding of predator-prey interactions, most theory developed surrounding predator dependence has been developed for predators that feed on only one prey. This leaves a large gap in our knowledge of the potential effects of predator dependence on predator-prey interactions. First, generalist predators can play an important role in mediating the coexistence among prey (Paine 1966, Levine 1976, Holt 1977). For example, generalist predators generate indirect effects among prey that can alter their coexistence (Holt 1977). Even if prey do not directly compete with one another, predation generates apparent competition among prey because the positive effects of a focal prey species on predators lead to greater negative effects of the predator on alternative prey. If apparent competition is strong enough, it can lead to the exclusion of alternative prey (The $\mathrm{P}^{*}$ rule; Holt et al. 1994). Generalist predators also can mediate coexistence among prey that directly compete through keystone predation (Paine 1966, Vance 1978, Holt et al. 1994, Leibold 1996). Keystone predation occurs when the competitively dominant prey is more susceptible to predation than alternative prey. Under this scenario, predation on the dominant competitor can allow for the persistence of prey that would be competitively excluded in the absence of the predator. The outcomes of both apparent competition and 
keystone predation are dependent on the strength of the predator-prey interactions within the system. As predator dependence alters the strength of predatorprey interactions, predator dependence is expected to alter the circumstances under which we expect prey to coexist.

Another reason predator-dependent functional responses are likely to have important consequences for generalist predator-prey interactions is that predator dependence generates density dependence within the predator population. Previous theory has shown that density dependence in predators can have important community-level effects. For example, Abrams and Matsuda (1996) have shown that density dependence in predators can reverse apparent competition by leading to positive indirect effects among prey or apparent mutualism. Apparent mutualism occurs because, when predators have saturating functional responses, prey not only have a negative indirect effect on one another through their positive effect on the consumer, they also have a positive indirect effect on one another because an increase in prey density leads to greater saturation of the predator's feeding rate.

When predators experience density dependence, the positive indirect effect of prey on one another can outweigh their negative indirect effects leading to an overall net positive effect of prey on one another. Although Abrams and Matsuda (1996) mainly focus on density-dependent mortality in predators, they also suggest that predator dependence in predator functional responses is unlikely to generate strong enough density dependence in predators to cause apparent mutualism.

However, they only consider one form of predator-dependent functional responses (the BeddingtonDeAngelis functional response). This raises the possibility that functional response models allowing for stronger density dependence in the consumer may allow for apparent mutualism. Altogether, these previous results suggest that predator-dependent functional responses are likely to have important effects in generalist predator-prey systems.

Here, we use one predator-two prey models incorporating predator-dependent functional responses to explore how the strength of predator dependence alters coexistence among prey and the overall net effects among prey (the effects of prey on one another after accounting for their direct and indirect interactions). To do so, we consider two predator functional response models: the Beddington-DeAngelis and Crowley-Martin functional responses. We also consider scenarios in which the prey do not interact directly and in which they directly compete with one another. We find that predator dependence can alter the coexistence outcomes among prey while also altering the strength and potentially sign of the net effects among prey. These results suggest that predator dependence is likely to be an important factor influencing the effects of generalist predators on the structure and dynamics of communities.

\section{Methods}

\section{Models}

We analyze standard ordinary differential equation models for the densities of a single predator, $P$, feeding on two resources, $R_{i}$, where $i=1$ and 2 for resource 1 and 2 respectively. When the resources do not interact directly, the dynamics of the resources are described by,

$\frac{d R_{i}}{d t}=r_{i} R_{i}\left(\frac{K_{i}-R_{i}}{K_{i}}\right)-P f_{i}\left(R_{1}, R_{2}, P\right)$

where $r_{i}$ is the intrinsic growth rate of resource $i, K_{i}$ is the carrying capacity of resource $i$, and $f_{i}\left(R_{1}, R_{2}, P\right)$ is the functional response of the predator on resource $i$. When the resources directly compete with one another, the resource dynamics are described by,

$\frac{d R_{i}}{d t}=r_{i} R_{i}\left(\frac{K_{i}-R_{i}-\alpha_{i j} R_{j}}{K_{i}}\right)-P f_{i}\left(R_{1}, R_{2}, P\right)$

where $a_{i j}$ is the per capita reduction of the growth rate of resource $i$ caused by resource $j$ relative to the effect of resource $i$ on its own growth rate and all other parameters are defined as above. Regardless of whether the prey directly interact, the dynamics of the predator are described as,

$\frac{d P}{d t}=P\left(\sum_{i=1}^{2}\left[e_{i} f_{i}\left(R_{1}, R_{2}, P\right)\right]-m\right)$

where $e_{i}$ is the conversion efficiency of resource $i$ into predators, $m$ is the per capita mortality rate of the predator, and all other parameters are defined as above.

\section{Predator functional responses}

To determine the effects of predator dependence on the coexistence of resources and their net effects on one another, we consider two predator-dependent functional responses designed to model mutual interference among predators. The first functional response we consider is the Beddington-DeAngelis functional response where,

$f_{i}\left(R_{1}, R_{2}, P\right)=\frac{a_{i} R_{i}}{1+\sum_{i=1}^{2}\left[a_{i} h_{i} R_{i}\right]+\gamma P}$

$a_{i}$ is the space clearance rate of the predator on prey $i$, $h_{i}$ is the time required for the predator to handle an individual of resource $i$, and $\gamma$ is the per capita interference rate among predators. We also consider the Crowley-Martin functional response where, 
$f_{i}\left(R_{1}, R_{2}, P\right)=\frac{a_{i} R_{i}}{1+\sum_{i+1}^{2}\left[a_{i} h_{i} R_{i}\right]+\sum_{i=1}^{2}\left[a_{i} h_{i} \gamma R_{i} P\right]+\gamma P}$

$\lambda$ is the per capita interference rate among predators, and all other parameters are defined above. The Crowley-Martin functional response models interference that occurs both when predators are searching and handling prey, whereas interference in the Beddington-DeAngelis functional response only occurs during the search process (Crowley and Martin 1989). These differences among the functional response qualitatively change how predator feeding rates respond to predator densities. In the Beddington-DeAngelis functional response, the effect of interference decreases as prey densities increase.

Specifically, in the limit of infinite prey density, predator density has no effect on the feeding rates of predators (Skalski and Gilliam 2001). In the limit, this is because all predators are occupied with handling prey and, thus, do not interfere with one another. In contrast, for the Crowley-Martin functional response, interference continues to affect predator feeding rates even at high prey densities due to interference occurring during the process of handling prey (Crowley and Martin 1989, Skalski and Gilliam 2001; Figure 1). In both forms, the functional response collapses to a standard Holling type II form when the interference parameter is set to zero (i.e. prey dependence only).

\section{Analytical methods}

Below, we explain the methods we used to examine the effects of predator dependence within the model on the coexistence among prey and their net effects on one another.

\section{Effects of predator dependence on coexistence among prey}

To evaluate how predator dependence influences the coexistence among prey, we examined a variety of scenarios using both the Beddington-DeAngelis and Crowley-Martin functional responses. For the case in which the prey do not directly compete (Equation 1), we examined the effects of predator dependence on coexistence when: 1) the prey coexist in the presence of the predator and in the absence predator dependence, and 2) one prey is excluded by the other via apparent competition in the absence of predator dependence. For the case in which the prey directly compete (Equation 2), we examined the effects of predator dependence when: 1) the prey coexist regardless of the presence of the predator, 2) the prey coexist in the absence of the predator, but one prey is excluded by the other via apparent competition in the presence of the predator, and 3) one prey competitively excludes the other

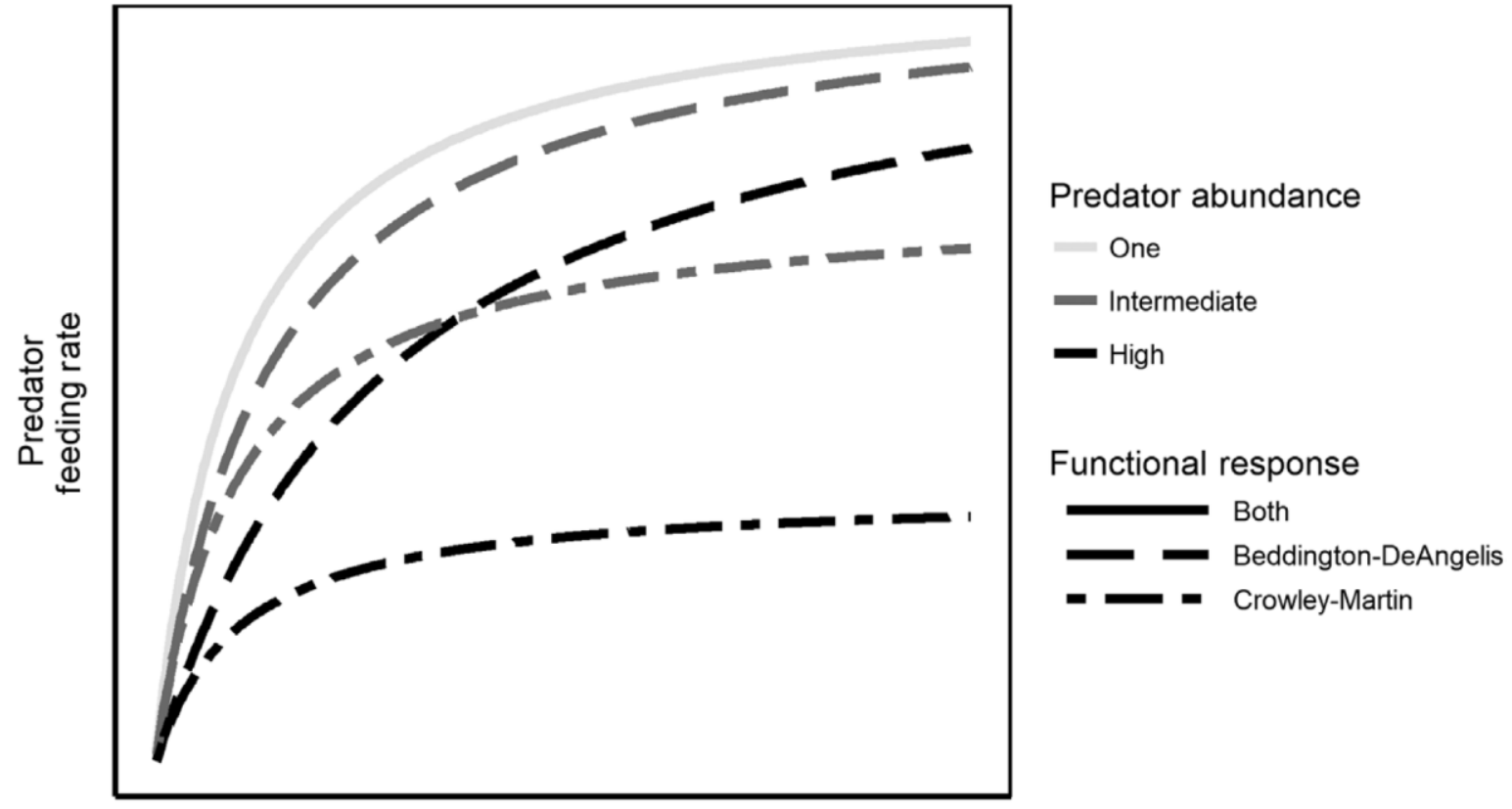

Prey density

Figure 1. The Beddington-DeAngelis and Crowley-Martin functional responses differ qualitatively in the relationship between predator density and the predator feeding rate at high prey densities. Under the Beddington-DeAngelis functional response (dashed lines), the effect of predator density (darker shades of gray) on the predator feeding rate decreases at high prey densities converging to the feeding rate in the absence of interference (lightest gray, solid line). In contrast, under the Crowley-Martin functional response (dotdashed lines), the effect of predator density (darker shades of gray) on the predator feeding rate remains at high prey densities. 
in the absence of predation, but the predator facilitates coexistence in the absence of predator dependence (keystone predation).

In the scenarios in which predator dependence altered coexistence among the prey, we examined how the strength of predator dependence altered the ability of the excluded prey to invade the system consisting of the predator and the non-focal prey. The invasibility of the system defines whether the prey are able to coexist with one another and is given by the per capita growth rate of the focal species while the other species in the system are at equilibrium (MacArthur and Levins 1967, Chesson 2000).

\section{Effects of predator dependence on the net effects among prey}

In the analyses of the effects of predator dependence on the coexistence among prey, we also examined the effects of predator dependence on the preys' net effects on one another. To do so, we used theory developed on the effects of press perturbations (Bender et al. 1984, Novak et al. 2016). Press perturbations, as opposed to pulse perturbations, are sustained or chronic perturbations to a system. Theory has shown that in the context of press perturbations, one can calculate the net effects among species by considering how a sustained press perturbation to one species will influence the equilibrium densities or growth rates of the other species in the system (Bender et al. 1984, Novak et al. 2016). Specifically, we examine the net effects among prey using the normalized net effects matrix introduced by Novak et al. (2016). The normalized net effects matrix is an nxn matrix where $\mathrm{n}$ is the number of species in the system. The $i, j$ th entry of the net effects matrix describes the expected change in the abundance of species $i$ given a sustained one unit increase in the population size of species $j$. This matrix is calculated by taking the negative inverse of the Jacobian of the system evaluated at the equilibrium and then normalizing each $i, j$ th entry by the $j, j$ th entry (the effect of species $j$ on itself; Novak et al. 2016).

For the parameter values for which the two prey species coexisted within the coexistence analyses, we calculated the normalized net effects matrix and examined how the entries corresponding to the effects of prey 1 on prey 2 and prey 2 on prey 1 changed with changes in the magnitude of predator dependence.

We were unable to obtain analytical results given the complexity of the models. Therefore, we used numerical analyses in Mathematica (ver. 12.0.0.0). The effects of predator dependence on the coexistence among prey and their net effects on one another were qualitatively similar under a wide range of parameter values. The Mathematica code used in the analyses can be found at: doi: 10.5281 / zenodo.3838267.

\section{Results}

Effects of predator dependence on coexistence among prey

The effects of predator dependence on the coexistence among prey are dependent on whether the prey directly compete with one another and the role that the predator plays in determining coexistence among prey in the absence of predator dependence (Figure 2A). In addition, the effects of predator dependence on coexistence can largely be understood as a consequence of the weakening effects of predator dependence on predator-prey interactions.

For the cases in which prey do not directly interact and in which the prey compete but coexist in the absence of the predator, predator dependence either has no effect on coexistence among the prey or can facilitate coexistence. If the prey coexist with one another and the predator in the absence of predator dependence, predator dependence does not influence the coexistence of the prey (Figures 3,4). If the prey do not coexist in the absence of predator dependence due to one prey excluding the other via apparent competition, predator dependence can facilitate coexistence among the prey (Figure 5, only the case with no direct interactions among prey is shown). This occurs because predator dependence weakens the predator-prey interactions reducing the positive effect of the superior apparent competitor on the predator and thus weakening apparent competition and preventing exclusion of the inferior apparent competitor. When prey compete directly and one prey would competitively exclude the other but the predator allows the prey to coexist, predator dependence can hinder coexistence (Figure 6). Under this scenario, the prey are able to coexist because the negative effect of the predator on the superior direct competitor prevents competitive exclusion of the inferior direct competitor. As predator dependence weakens this interaction, predator dependence undermines the predator's ability to foster coexistence among the prey (Figure 6). The effects of predator dependence on the coexistence among prey are the same for the Beddington-DeAngelis and Crowley-Martin functional responses with the exception that the effects occur at lower interference rate values for the Crowley-Martin functional response for the same values of the other model parameters (Figures 5, 6). 

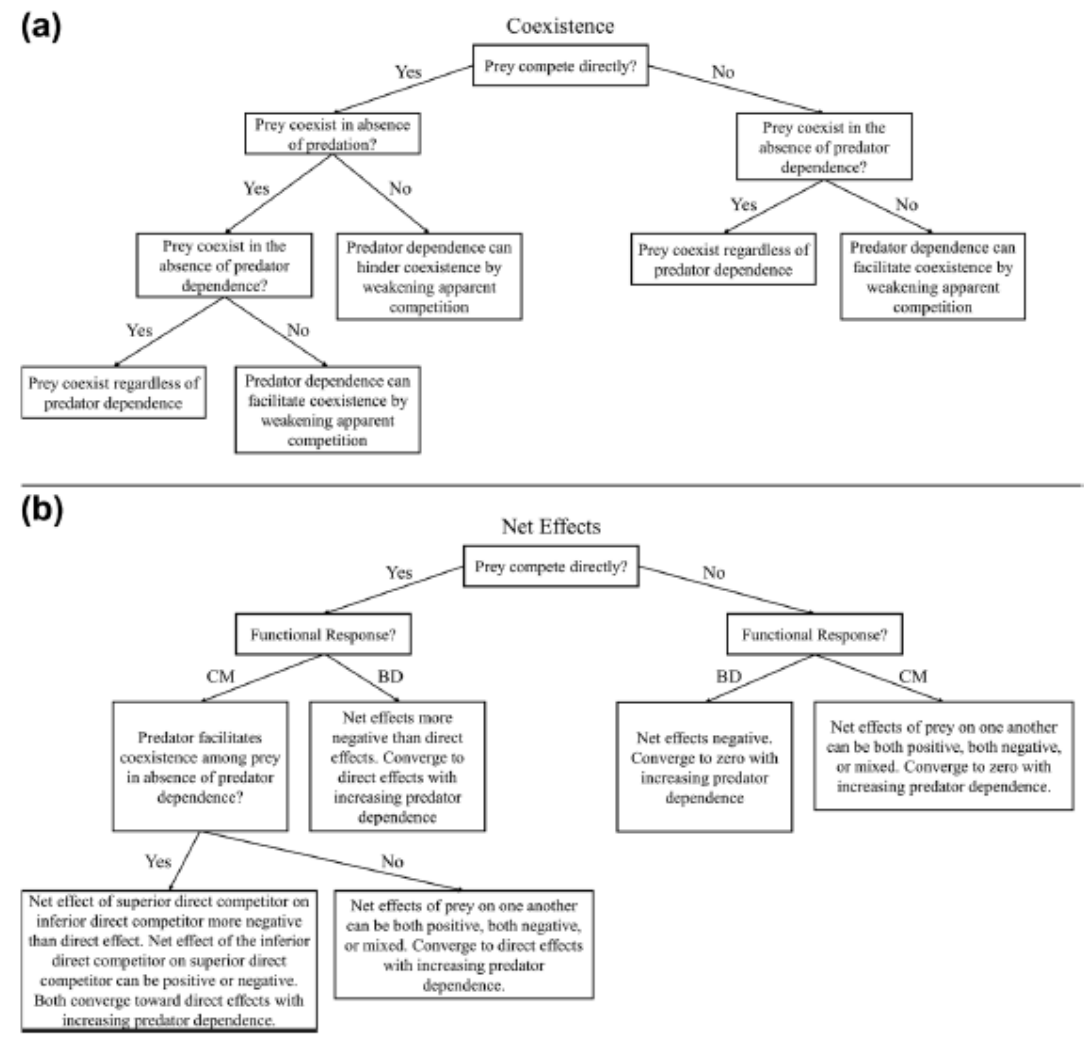

Figure 2. Decision trees describe how predator dependence alters: 1) coexistence among prey depending upon whether the prey compete directly and the role of the predator in determining coexistence in the absence of predator dependence (a), and 2) the net effects of prey on one another depending on the functional response exhibited by predators $(\mathrm{BD}=\mathrm{Beddington}-\mathrm{De} A n g e l i s, \mathrm{CM}=$ Crowley-Martin), predator effects on coexistence, and parameter values (b).

\section{Effects of predator dependence on the net effects among prey}

In all the scenarios considered, increasing predator dependence causes the net effects among prey to converge to their direct effects (Figure 2B). When the prey do not directly interact, the net effects of the prey on one another converge towards zero. Whereas, when the prey directly compete with one another, the net effects of the prey on one another converge to their direct competitive effects. This occurs because predator dependence weakens the predator-prey interactions generating the indirect effects among the prey.

The behavior of the net effects with increasing predator dependence before converging to the preys direct effects depends on the functional response exhibited by the predators (Figure 2B). If the predator exhibits a Beddington-DeAngelis functional response, the net effects of prey on one another are more negative than the direct effects of the prey on one another in the absence of predator dependence and approach the direct effects of the prey on one another from below with increasing predator dependence (Figures 3B, $4 \mathrm{~B}, 5 \mathrm{C}, 6 \mathrm{C})$. If the predator exhibits a Crowley-Martin functional response, the effects of predator dependence on the net effects among prey can be more complex. In all of the cases except that in which one prey would competitively exclude the other in the absence of the predator, the net effects of prey on one another can remain negative (Figure 3B), both be positive (Figure $5 \mathrm{C}$ ), or can be mixed (Figure $4 \mathrm{~B}$ ) before converging to the preys' direct effects on one another, depending on the parameter values of the model. In general, positive net effects among prey occur under parameter values where the predator-prey interactions are strong and the predator is saturated in its ability to respond to the press perturbation increase in prey. When the predator-prey interactions are weaker, the predator is not saturated and is able to increase its predation rate on the alternative prey when the focal prey's abundance is increased. In the case that one prey would competitively exclude the other in the absence of the predator, the net effect of the superior direct competitor remains negative and converges to the direct effect on the inferior direct competitor from below (Figure 6F). The net effect of the inferior direct competitor can be positive or negative before converging to the direct effect on the superior direct competitor depending on the parameter values. 
(a)
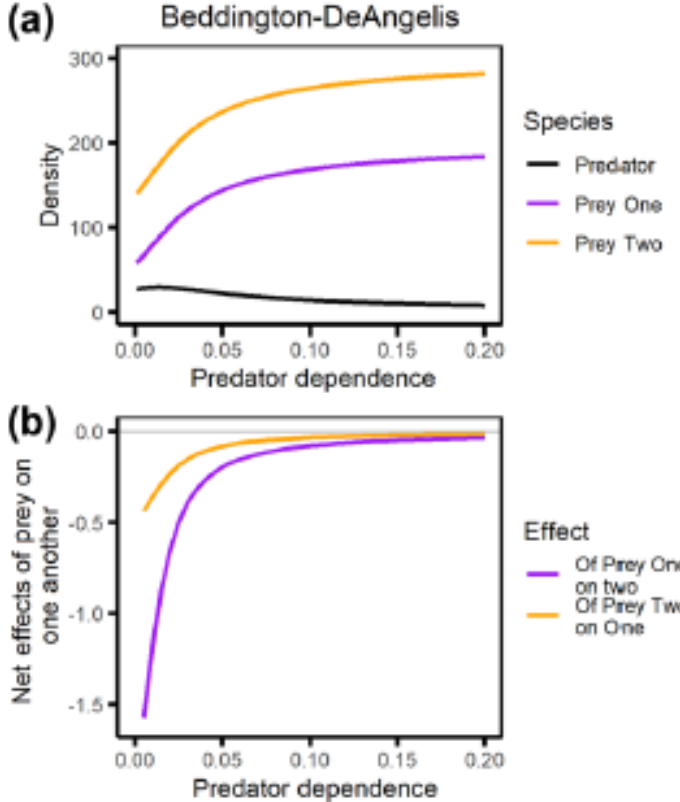

(c) Crowley-Martin
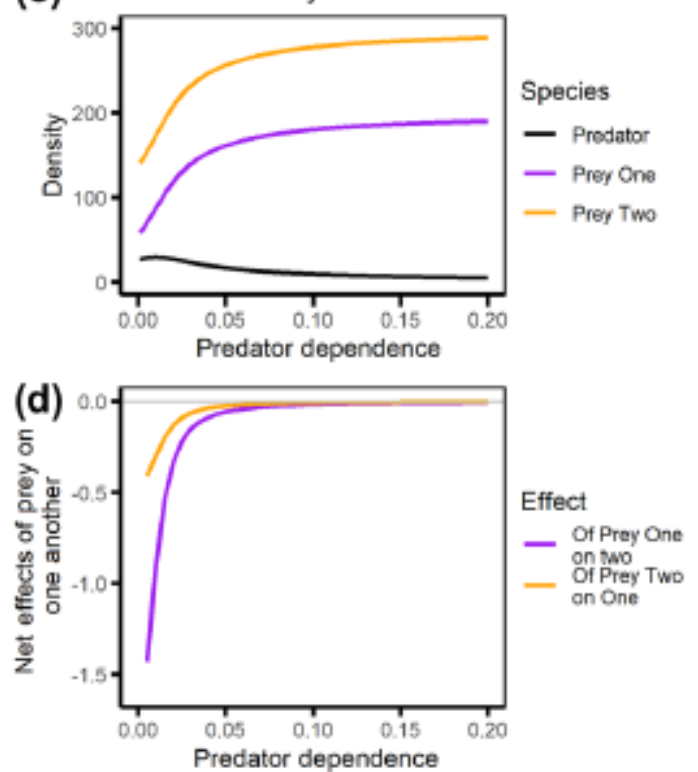

Figure 3. Effects of predator dependence on predator and prey densities $(a, c)$ and the net effects among prey $(b$ and $d)$ when prey do not compete directly and coexist in the absence of predator dependence. (a) and (b) illustrate densities and net effects when predators exhibit a Beddington-DeAngelis functional response. (c) and (d) illustrate densities and net effects when predators exhibit a CrowleyMartin functional response. Under alternative parameter values for the Crowley-Martin functional response, the net effects of prey on one another can be both positive, mixed, or both negative (as shown here) before converging to zero. Parameter values used to produce the figure were: $r_{1}=0.3, r_{2}=0.2, K_{1}=200, K_{2}=300, a_{1}=0.01, a_{2}=0.005, \eta_{1}=\eta_{2}=0.2, e_{1}=e_{2}=0.2, m=0.2$.

(a)
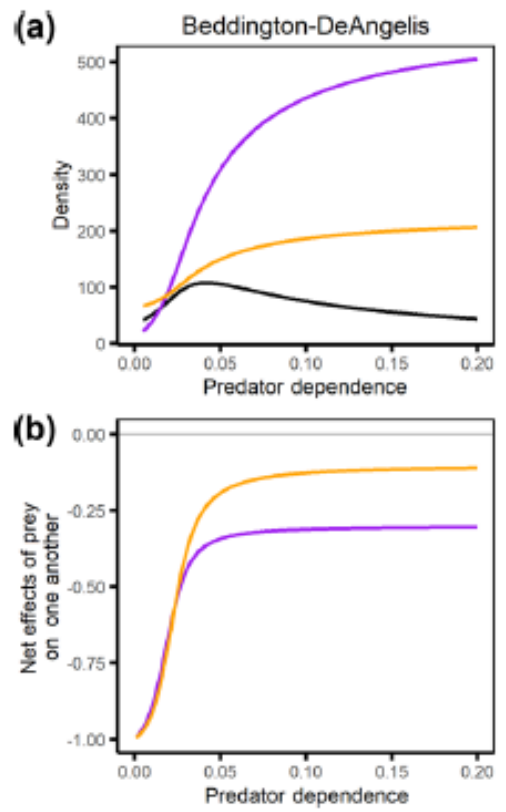

(c)
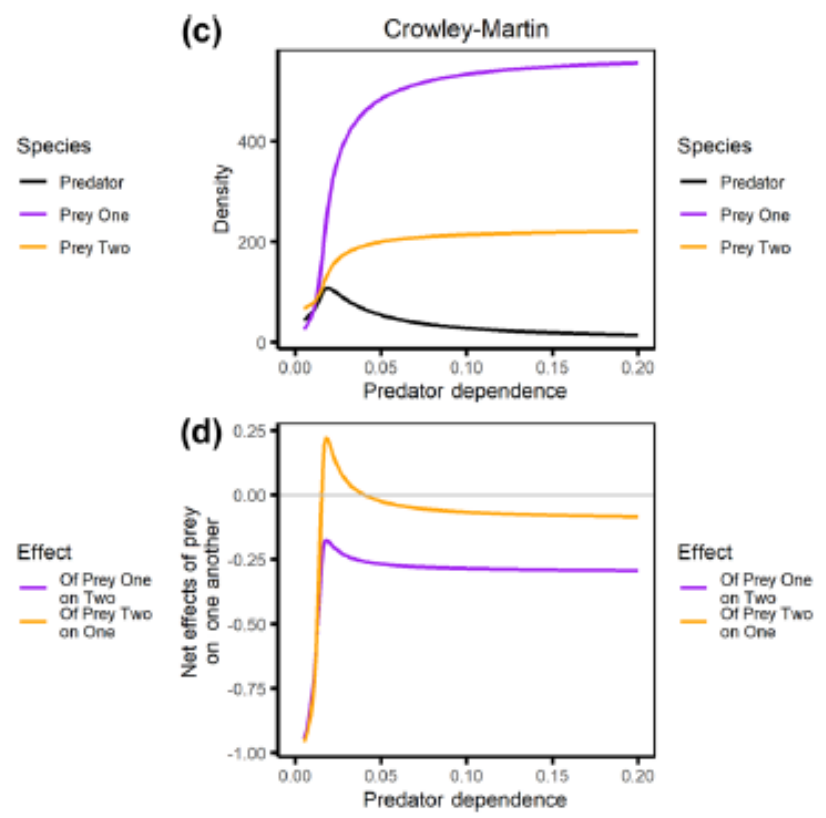

Figure 4. Effects of predator dependence on predator and prey densities ( $a$ and c) and the net effects among prey (b and d) when prey directly compete with one another but coexist in the presence of the predator and the absence of predator dependence. (a) and (b) illustrate densities and net effects when predators exhibit a Beddington-DeAngelis functional response. (c) and (d) illustrate densities and net effects when predators exhibit a Crowley-Martin functional response. Under alternative parameter values for the CrowleyMartin functional response, the net effects of prey on one another can be both positive, mixed (as shown here), or both negative before converging to the direct competitive effects of prey on one another. Parameter values used to produce this figure were: $r_{1}=0.3, r_{2}=$ $0.35, K_{1}=600, K_{2}=400, a_{1}=a_{2}=0.01, a_{21}=0.3, a_{12}=0.1, \eta_{1}=\eta_{2}=0.3, e_{1}=e_{2}=0.5, m=0.3$. 

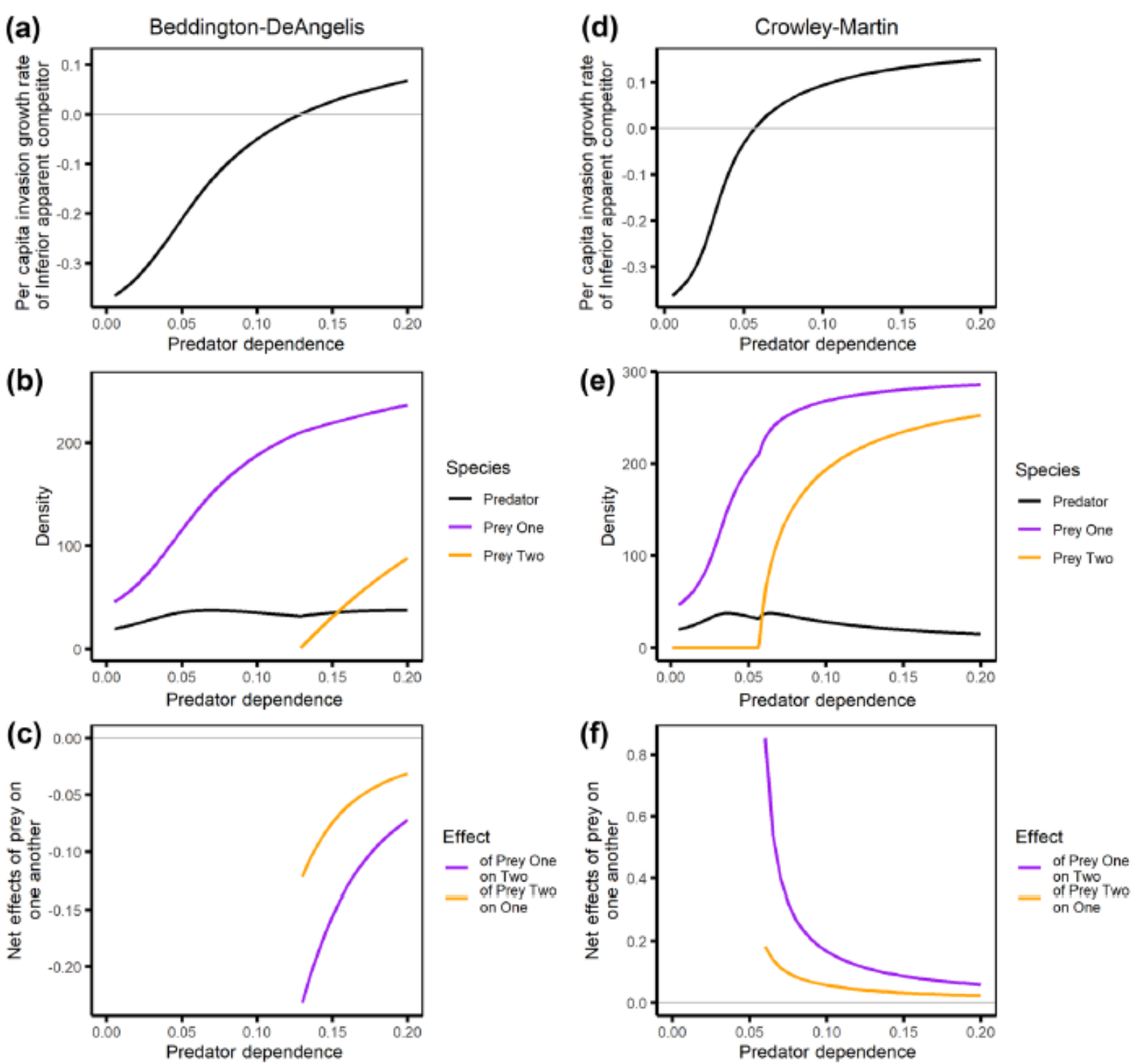

Figure 5. Effects of predator dependence on the invasibility of the system by the inferior apparent competitor (prey two; a and d), predator and prey densities ( $\mathrm{b}$ and e), and the net effects among prey ( $\mathrm{c}$ and $\mathrm{f}$ ) when the prey do not interact and the superior apparent competitor (prey one) excludes the inferior apparent competitor (prey two) in the absence of predator dependence. (a), (b), and (c) illustrate invasibility, densities, and net effects when predators exhibit a Beddington-DeAngelis functional response. (d), (e), and (f) illustrate invasibility, densities, and net effects when predators exhibit a Crowley-Martin functional response. Under alternative parameter values for the Crowley-Martin functional response, the net effects of prey on one another can be both positive (as shown here), mixed, or both negative before converging to zero. Parameter values used to produce this figure were: $r_{1}=0.5, r_{2}=0.2, K_{1}=K_{2}$ $=300, a_{1}=0.03, a_{2}=0.04, \eta_{1}=\eta_{2}=0.2, e_{1}=e_{2}=0.5, m=0.5$.

\section{Discussion}

Predator dependent functional responses appear widespread. However, much of the theory on the effects of predator dependence has focused on its effects in specialist predator-prey pairs, linear food chains, or inter-specific interactions among predators (Beddington 1975, DeAngelis et al. 1975, Arditi and Ginzburg 1989, Arditi and Ginsburg 2012, McPeek and Siepielski 2019). It has been unclear, however, what role predator dependence may play in generalist predator-prey interactions and food webs more generally, even though predator dependence occurs in some predators foraging on broad diets (Novak et al. 2017). Here, using a one predator-two prey model incorporating predator dependent functional responses, we show that: 1) predator dependence can either facilitate, hinder, or have no effect on coexistence among prey depending on whether the prey compete directly and the predator's role in mediating coexistence in the absence of 
(a)

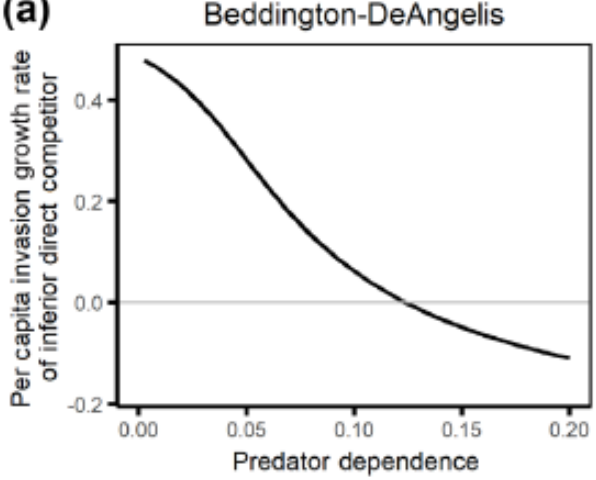

(b)

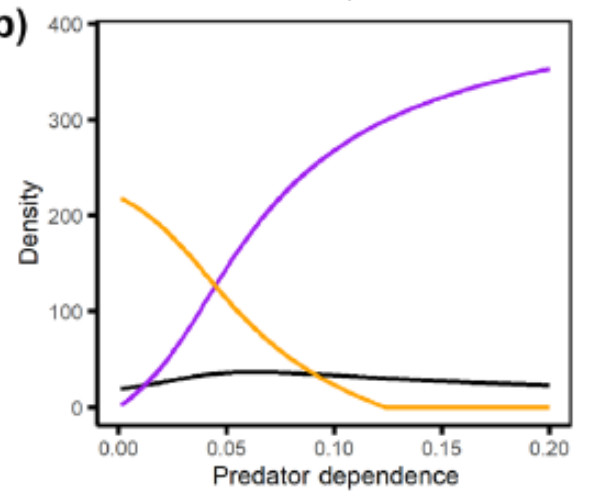

(c)

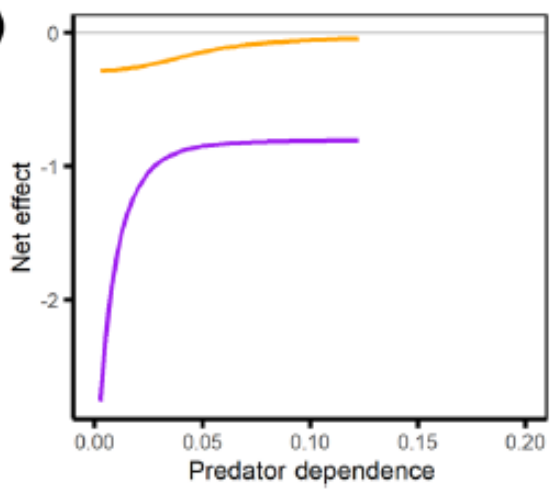

(d)

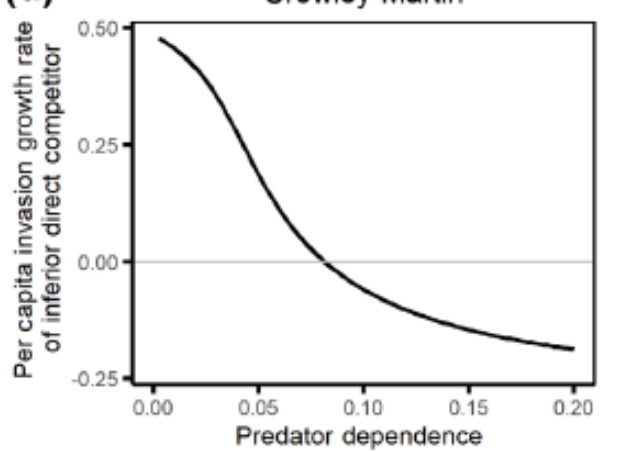

(e)
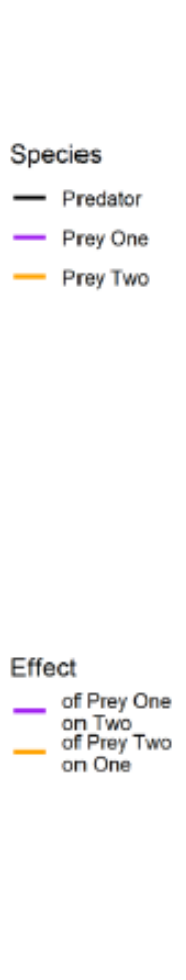

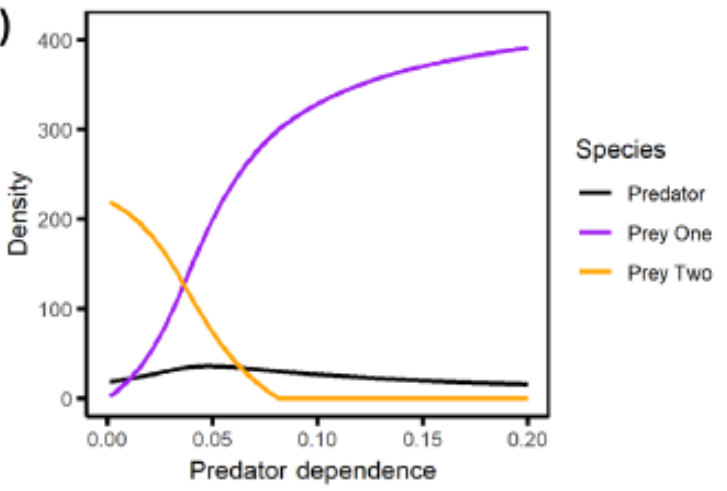

(f)

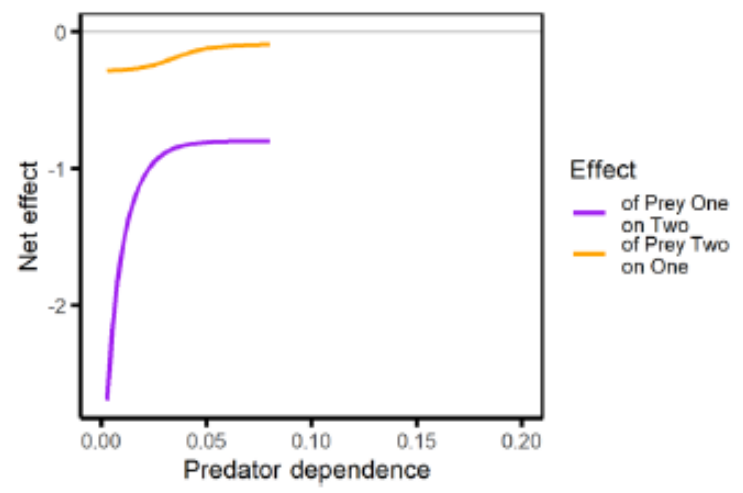

Figure 6. Effects of predator dependence on the invasibility of the system by the inferior direct competitor (prey two; a,d), predator and prey densities $(\mathrm{b}, \mathrm{e})$, and the net effects among prey $(\mathrm{c}, \mathrm{f})$ when the prey directly compete, one prey would exclude the other in the absence of predation, and the predator facilitates coexistence in the absence of predator dependence. (a), (b), and (c) illustrate invasibility, densities, and net effects when predators exhibit a Beddington-DeAngelis functional response. (d), (e), and (f) illustrate invasibility, densities, and net effects when predators exhibit a Crowley-Martin functional response. Under alternative parameter values for the Crowley-Martin functional response, the net effects of the inferior direct competitor (prey two) can be positive before converging towards the direct competitive effect on the superior direct competitor (prey one). Parameter values used to produce this figure were: $r_{1}=0.3, r_{2}=0.75, K_{1}=450, K_{2}=250, a_{1}=0.0175, a_{2}=0.005, a_{21}=0.8, a_{12}=0.1, \eta_{1}=\eta_{2}=0.1, e_{1}=e_{2}=0.5, m=0.5$.

predator dependence, and 2) predator dependence generally weakens the net effects of prey on one another and can lead to positive net effects among prey in the case of the Crowley-Martin functional response. These results suggest that predator dependence in generalists may have several consequences for the structure and dynamics of communities by altering the coexistence among prey and the net effects of species on one another.
Our results suggest that predator dependence can alter the role of predators in determining coexistence among prey because of the effect of predator dependence on overall interaction strengths. Whether or not prey directly compete with one another, if one prey would exclude the other via apparent competition in the absence of predator dependence, predator dependence can facilitate the coexistence of the prey. In contrast, when prey compete and the predator facilitates 
coexistence among prey through keystone predation, predator dependence can hinder coexistence among prey. Thus, the overall effects of predator dependence in communities could increase, decrease, or have equivocal effects on the diversity of prey depending on the patterns of interactions among prey. In one previous food web study, Rall et al. (2008) showed that predator dependence in allometric food web models had a positive, saturating effect on the richness of species in the food web. The authors attribute the positive effect of predator dependence on species richness to the weakening of the predator-prey interactions and thus increased persistence of species with greater predator dependence.

Our results suggest, however, that stronger predator dependence and weakening predator-prey interactions should also lead to extinctions of prey if the predators otherwise facilitate coexistence among competing prey. It is possible that the methods used by the authors, particularly the assumption that predators' preferences among prey are uniform, might have prevented this effect, as predator facilitation of coexistence among competing prey requires that the inferior direct competitors are less susceptible to predation (Vance 1978, Holt et al. 1994, Leibold 1996). Another potential explanation for the lack of a negative effect of predator dependence on species richness is that all of the consumers in the model exhibit predator dependence (Rall et al. 2008). Thus, predator dependence within the competing prey themselves may be strong enough to prevent the possibility of competitive exclusion among them. Regardless, these issues highlight some of the difficulties that must be overcome to understand the role that predator dependence may play in shaping food web dynamics. For example, is predator dependence expected to be stronger at different trophic levels? Would different patterns of predator preference other than uniform lead to different predator dependence-species richness relationships? Future empirical and theoretical work is certainly necessary to uncover the potential role of predator dependence in complex food webs.

It is well known that predation can generate indirect effects among prey, particularly in the form of apparent competition (Holt 1977). Our results show that predator dependence, in general, is likely to weaken the indirect effects among prey. As predator dependence increases in the models, the net effects of prey on one another either converge towards zero when the prey do not directly interact or converge towards the direct effects of prey on one another when the prey compete. Furthermore, when the predator exhibits a Crowley-Martin functional response, the net effects among prey can exhibit a variety of patterns from apparent competition, to apparent mutualism (positive effects of prey on one another), and apparent predation (positive and negative reciprocal effects among prey).
Previous theory has shown that a variety of mechanisms such as density dependent predator mortality and adaptive predator or prey behavior can produce positive or mixed indirect effects among prey (Abrams 1987, Abrams and Matsuda 1996). Our results suggest that the strong saturating effect of predator dependence as occurs in the Crowley-Martin functional response can be added to the list of mechanisms capable of producing positive indirect effects among prey sharing a predator. Furthermore, although less well known than apparent competition, these various possible patterns of indirect effects such as apparent mutualism, predation, amensalism, and others have been observed empirically (Menge 1995, Chaneton and Bonsall 2000, Tack et al. 2011). Given the important influence of indirect effects on community structure and food web dynamics and their predictability (Bender et al. 1984, Yodzis 1988, Wootton 1994, Menge 1995, Montoya et al. 2009, Novak et al. 2011), and the widespread empirical documentation of predator dependence in both generalist and specialist predators (Skalski and Gilliam 2001, DeLong and Vasseur 2011, Novak et al. 2017), further attention should be given to the role predator dependence may play in altering the indirect effects among prey and their resultant consequences.

Predator dependence is generally stabilizing in specialist predator-prey interactions because it reduces interaction strengths as predators become more abundant, increasing the resiliency of systems to perturbations and dampening predator-prey cycles (Arditi et al. 2004). A linear stability analysis of the models presented here agrees with that conclusion (Supplementary material Appendix 1). However, our models also suggest that predator dependence can be viewed as destabilizing under different definitions of stability. For example, predator dependence can eliminate coexistence among prey when predators facilitate coexistence among directly competing prey; that is, changes in predator dependence may prevent keystone predation leading to extinctions. Furthermore, as previous studies have pointed out, strong predator dependence can be viewed as destabilizing by reducing predator densities to low levels (Arditi et al. 2004). With strong predator dependence, the resulting low densities of the predator can make the predator population susceptible to extinction due to stochastic population fluctuations, or if interference is linked to overall foraging, can eliminate a positive equilibrium altogether (DeLong and Vasseur 2013). Therefore, predator dependence may be largely stabilizing in terms of increasing the resiliency of systems and yet simultaneously destabilizing in terms of increasing the risk of extinction if predators facilitate coexistence among competing prey or if predator dependence leads to low densities of the predator. These results highlight the multifaceted nature of stability and that ecological processes can be stabilizing or destabilizing depending on the circumstances and 
facet of stability one is interested in (Ives and Carpenter 2007, Arnoldi et al. 2016, Donohue et al. 2016).

Despite the results of our models illustrating that predator dependence can alter the strength of indirect effects and coexistence among prey, it is difficult to assess this theory in light of existing empirical research. First, the magnitude of predator dependence used in our models is well within the observed range of predator dependence quantified in experiments (Skalski and Gilliam 2001). However, these experiments have generally measured predator dependence under laboratory conditions with only a single prey available. In one study that quantified predator dependence in a generalist predator under field conditions with its full suite of prey available, Novak et al. (2017) found that predator dependence in intertidal whelks was weak but detectable. Whether interference typically is weak in generalist predators in realistic conditions is still unknown. In this same study, Novak et al. (2017) also showed that the strength of predator dependence in their system may be dependent on the relative densities of prey. This suggests that existing functional response models may not capture the complexity of predator dependence in generalist predators. Deriving functional responses capable of exhibiting variable predator dependence or designed specifically to model predator dependence in generalist predators may lead to different conclusions regarding the effects of predator dependence in generalist predator-prey systems. Lastly, our models were restricted to considering a predator feeding on only two prey species. As most food web studies have not included predator dependent functional responses, it remains unclear whether the conclusions reached here will apply in more species rich, reticulate food webs. The allometric food web study by Rall et al. (2008), however, provides a useful starting point.

Our results were overall similar between the two functional forms of predator dependence we used, but there were some qualitatively important differences. These differences suggest that the form of predator dependence determines the effect of predator dependence on food webs. The variety of predator dependent models available (Hassel and Varley 1969, Abrams 1982, Arditi and Ginsburg 1989, Tyutyunov et al. 2008) implicitly invoke different mechanisms that alter the functional response in different ways or model predator dependence phenomenologically. We have little information about the behavioral mechanisms generating predator dependence, making it difficult to choose or implement the most appropriate functional response models. For example, the Beddington-DeAngelis and Crowley-Martin functional responses used here model predator dependence as the product of mutual interference among predators (Beddingtion 1975, DeAngelis et al. 1975, Crowley and Martin 1989). However, predator dependence in functional responses can also be the product of prey rather than predator behavior. Prey behavior such as adaptive anti-predator defenses and refuge use can lead to functional responses with functional forms differing from those modeling predator dependence through predator behavior (Charnov et al. 1976, Abrams 1992). Therefore, predator dependence generated through prey behavior may lead to different conclusions regarding the effects of predator dependence on prey coexistence and net effects. In general, continued research on the behavioral mechanisms underlying predator dependence will improve our understanding of the ecological role of predator dependence.

\section{Conclusions}

Nearly every study seeking to measure predator dependence in predator functional responses has found some level of predator dependence (Skalski and Gilliam 2001, DeLong and Vasseur 2011, Arditi and Ginsburg 2012). Previous theoretical and empirical research has shown that this predator dependence can have important ecological consequences on the stability of predator-prey systems (Arditi et al. 2004), the responses of food chains to enrichment (Arditi and Ginsburg 1989), and the invasion biology of predators (Griffen and Delaney 2007). Here we show that predator dependence in generalist predators can also influence the strength and potentially sign of the net effects that prey have on one another and their coexistence with one another. These results suggest that if predator dependence is widespread, it may have a variety of important effects on the role that predators play in determining the structure and dynamics of communities and food webs. Determining whether this is the case will require further empirical and theoretical research on the strength and form of predator dependence in generalist predators and the consequent impacts on the communities in which they are embedded.

Data availability statement - Data are available from the Github Digital Repository: doi:10.5281/zenodo.3838267 (Coblentz and DeLong 2020).

Funding - This project was supported by a James S. McDonnell Foundation grant to JPD.

Author contributions - Both authors designed the research. KEC developed and analyzed the models and wrote the first draft of the manuscript. Both authors contributed to revisions. Conflicts of interest - The authors declare no conflicts of interest.

\section{References}

Abrams, P. A. 1982. Functional responses of optimal foragers. Am. Nat. 120: 382-390.

Abrams, P. A. 1987. Indirect interactions between species that share a predator: varieties of indirect effects. In W. C. Kerfoot and A. Sih (eds.), Predation: Direct and Indirect Impacts on Aquatic Communities., pp. 38-54. University Press of New England. 
Abrams, P. A. 1992. Predators that benefit prey and prey that harm predators: unusual effects of interacting foraging adaptation. Am. Nat. 140: 573-600.

Abrams, P. A. and Matsuda, H. 1996. Positive indirect effects between prey species that share predators. Ecology 77: 610616.

Abrams, P. A. and Ginzburg, L. R. 2000. The nature of predation: prey dependent, ratio dependent or neither? Trends Ecol. Evol. 15: 337-341.

Arditi, R. and Ginzburg, L. R. 1989. Coupling in predatorprey dynamics: ratio-dependence. J. Theor. Biol. 139: 311326.

Arditi, R. and Ginzburg, L. R. 2012. How Species Interact: Altering the Standard View on Trophic Ecology. Oxford University Press.

Arditi, R. et al. 2004. Does mutual interference always stabilize predator-prey dynamics? A comparison of models. $C R$ Biol. 327: 1,037-1,057.

Arnoldi, J. et al. 2016. Resilience, reactivity, and variability: a mathematical comparison of ecological stability measures. J. Theor. Biol. 389: 47-59.

Beddington, J. R. 1975. Mutual interference between parasites of predators and its effect on searching efficiency. J. Anim. Ecol. 44: 331-340.

Bender, E. A. et al. 1984. Perturbation experiments in community ecology: theory and practice. Ecology 65: 1-13.

Chaneton, E. J. and Bonsall, M. B. 2000. Enemy-mediated apparent competition: empirical patterns and evidence. Oikos 88: 380-394.

Charnov, E. L. et al. 1976. Ecological implications of resource depression. Am. Nat. 110: 247-259.

Chesson, P. 2000. Mechanisms of maintenance of species diversity. Annu. Rev. Ecol. Evol. Syst. 31: 343-366.

Coblentz, K. E. and DeLong, J. P. 2020. Data from: Predatordependent functional responses alter the coexistence and indirect effects among prey that share a predator. Github Digital Repository, doi: 10.5281/zenodo.3838267.

Crowley, P. H. and Martin, E. K. 1989. Functional responses and interference within and between year classes of a dragonfly population. J. N. Am. Benthol. Soc. 8: 211-221.

DeAngelis, D. L. et al. 1975. A model for trophic interaction. Ecology 56: 881-892.

DeLong, J. P. and Vasseur, D. A. 2011. Mutual interference is common and mostly intermediate in magnitude. $B M C$ Ecol. 11: 1.

DeLong, J. P. and Vasseur, D. A. 2013. Linked exploitation and interference competition drives the variable behavior of a classic predator-prey system. Oikos 122: 1,393-1,400.

Donohue, I. et al. 2016. Navigating the complexity of ecological stability. Ecol. Lett. 19: 1,172-1,185.

Griffen, B. D. and Delaney, D. G. 2007. Species invasion shifts the importance of predator dependence. Ecology 88: 3012-3021.

Hassel, M. P. and Varley, G. C. 1969. New inductive population model for insect parasites and its bearing on population control. Nature 223: 1,133-1,136.

Holling, C. S. 1959. The components of predation as revealed by a study of small mammal predation of the European pine sawfly. Can. Entomol. 91: 293-320.

Holt, R. D. 1977. Predation, apparent competition, and the structure of prey communities. Theor. Popul. Biol. 12: 197229.

Holt, R. D. et al. 1994. Simple rules for interspecific dominance in systems with exploitative and apparent competition. Am. Nat. 144: 741-771.
Ives, A. R. and Carpenter, S. R. 2007. Stability and diversity of ecosystems. Science 317: 58-62.

Leibold, M. A. 1996. A graphical model of keystone predators in food webs: trophic regulation of abundance, incidence, and diversity patterns in communities. Am. Nat. 147: 784-812.

Levine, S. H. 1976. Competitive interactions in ecosystems. Am. Nat. 110: 903-910.

MacArthur, R. and Levins, R. 1967. The limiting similarity, convergence, and divergence of coexisting species. Am. Nat. 101: 377-385.

McPeek, M. A. and Siepielski, A. M. 2019. Disentangling ecologically equivalent from neutral species: the mechanisms of population regulation matter. J. Anim. Ecol. 88: 1,7551,765 .

Menge, B. A. 1995. Indirect effects in marine rocky intertidal interaction webs: patterns and importance. Ecol. Monogr. 65: 21-74.

Montoya, J. M. et al. 2009. Press perturbations and indirect effects in real food webs. Ecology 90: 2,426-2,433.

Novak, M. et al. 2011. Predicting community responses to perturbations in the face of imperfect knowledge and network complexity. Ecology 92: 836-846.

Novak, M. et al. 2016. Characterizing species interactions to understand press perturbations: what is the community matrix? Annu. Rev. Ecol. Evol. Syst. 47: 409-432.

Novak, M. et al. 2017. Quantifying predator dependence in the functional response of generalist predators. Ecol. Lett. 20: 761-769.

Paine, R. T. 1966. Food web complexity and species diversity. Am. Nat. 100: 65-75.

Rall, B. C. et al. 2008. Food-web connectance and predator interference dampen the paradox of enrichment. Oikos 117: 202-213.

Skalski, G. T. and Gilliam, J. F. 2001. Functional responses with predator interference: viable alternatives to the Holling type II model. Ecology 82: 3083-3092.

Solomon, M. E. 1949. The natural control of animal populations. J. Anim. Ecol. 18: 1-35.

Tack, A. J. M. et al. 2011. Can we predict indirect interactions from quantitative food webs? An experimental approach. J. Anim. Ecol. 80: 108-118.

Tyutyunov, Y., Titova, L. and Arditi, R. 2008. Predator interference emerging from trophotaxis in predator-prey systems: an individual-based approach. Ecol. Complex. 5: 4858.

Vance, R. R. 1978. Predation and resource partitioning in one predator-two prey model communities. Am. Nat. 112: 787813.

Wootton, J. T. 1994. The nature and consequences of indirect effects in ecological communities. Annu. Rev. Ecol. Evol. Syst. 25: 443-466.

Yodzis, P. 1988. The indeterminacy of ecological interactions as perceived through perturbation experiments. Ecology 69: 508-515.

Supplementary material (Appendix 1) is available online at https:// www.oikosjournal.org/appendix/oik-07309 and https://www.oikosjournal.org/sites/oikosjournal.org/files/appendix/oik-07309.pdf. 